View Article Online / Journal Homepage / Table of Contents for this issue

MARCET : APPLIOATION OF PETTENKOFER'S PROCESS.

493

XXXVIII.-On the Mode of Application of Pettenkofer's Process for the Determination of Carbonic Acid in Expired Air.

By William Marcet, M.D., F.R.S., F.C.S.

SinCE the year 1875, my attention has been directed in the summer time to the influence of altitude on the phenomena of respiration, and results obtained have been published in an abstract form in the Proceedings of the Royal Society for 1878 and 1879 (see also this Journal, 38, 483).

The first of these papers contains an account of the process I have adopted for the determination of the carbonic acid in expired air; a process which, although yielding trustworthy results after the introduction of a certain correction, was open to improvement. I have now succeeded in perfecting the mode of analysis, which in its present form yields correct and trustworthy results without any correction; while at the same time the apparatus required for that purpose is of a portable description.

The principle of the process is that of Pettenkofer's, the analysis being made by effecting the combination of the $\mathrm{CO}_{2}$ in a known bulk of air with a solution of barium hydrate of known strength, and ascertaining afterwards volumetrically the amount of barium left in solution by means of oxalic acid.

After experimenting with vessels of various forms, in which the combination of the carbonic acid with barium hydrate might be effected, the simplest in shape was adopted-a hollow glass cylinder, or wide tube, holding about 1,500 c.c. and closed at both ends by india-rubber corks. This cylinder fulfilled a double object, first that of measuring out a certain bulk of the air to be submitted to analysis, and secondly that of enabling the air to be treated with the 
normal solution of barinm. A sketch of the apparatus I used is figured in my first paper (Proceedings Royal Society, 1878).

The air was collected from the lungs into a strong india-rubber bag of a capacity measured under a certain pressure, determined by a water-gauge, and which capacity varied, one bag holding $39 \cdot 3$ and another 68.4 litres under a pressure of 1 inch of water. The cylinder was filled with air from the bag, being fixed in the upright position to a light tripod, similar to those employed by photographers. In the experiments already published, the air was drawn into the tube by means of water; a solution of common salt, in order to lose no carbonic acid by absorption, had served the purpose instead of water in the first instance, but on trying water, and then testing it, I found it absorbed no carbonic acid. An objection to this method arose from the circumstance that the tube invariably retained a certain quantity of water, which adhered to the inside of the glass, and became mixed with the 100 c.c. of the alkaline solution used for the analysis. The results obtained might fairly, it is true, bear a mutual comparison, as the volume of water retained in the tube was nearly the same in every case, as ascertained experimentally, but they were really all too high; a correction therefore became necessary, which was calculated by determining the mean volume of water retained in the tubs, and then taking into account the dilution of the normal solution arising from the admixture of this water with it. The correction was applied to all the determinations reported in my second paper, relating to work done on the Island and Peak of Teneriffe, while the estimations of $\mathrm{CO}_{2}$ made previously were also corrected and introduced into the second paper under the form of means, so that they might be compared with the mean results from the experiments made at Teneriffe.

This last summer the use of water with the object of aspiring the air from the bag into the cylinder has been entirely given up, and I have had the satisfaction of working with an apparatus which will be considered, I think, free from objections, the present arrangement being available, not only for the determination of carbonic acid in air expired, but also in various other cases.

The hollow cylinder or tube I now employ is made of very thick glass, ground at both ends and closed by two round discs or slabs also consisting of very thick glass ground on the side in contact with the tube. Brass pieces are fitted over the ends of the cylinder, made to adhere firmly to the glass and bearing a thread, allowing of a brass ring to be screwed down tightly over them. The brass rings are flattened out at the top so as to keep the glass discs firmly down on the ends of the cylinder; a small quantity of grease previously applied to the rim of the tube secures an air-tight joint. As will be presently explained, a vacuum is produced in this cylinder by means of a 


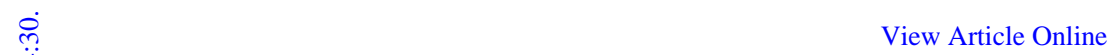

OF PETTENKOFER'S PROCESS, ETC.

common one-barrel air-pump, and by opening a stop-cock, air from the bag is let into the tube ; a repetition of this same operation ensures the perfect filling of the cylinder with air from the bag.

Apparates for the Analysis of Air Expired.

A

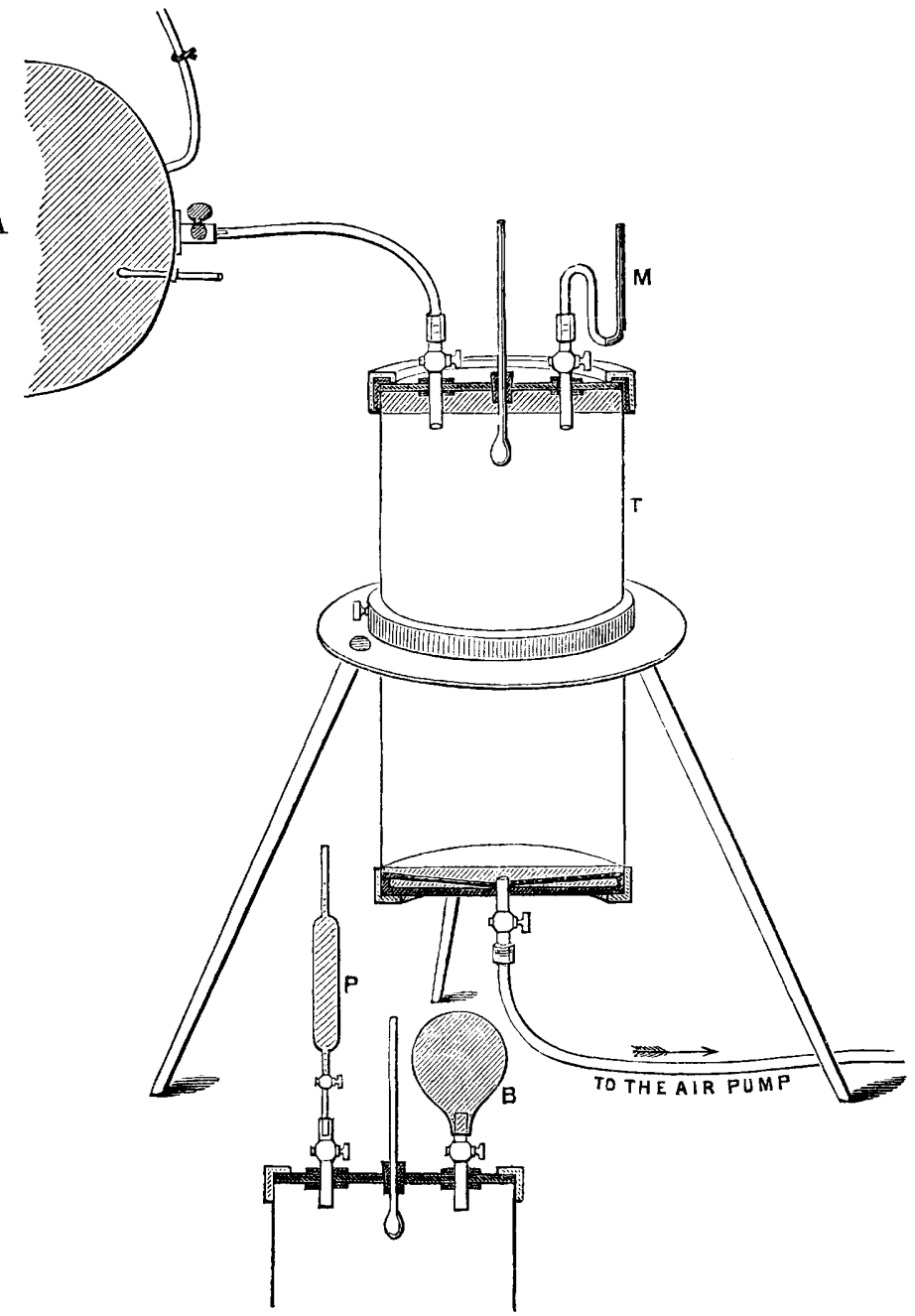

A. Bag holding the air to be analysed.

M. Manometer.

T. Tube in which the air is treated with the solution of barium.

B. Vulcanized india-rubber bag.

P. Pipette holding 100 c.c. of the solution of barium. 
I shall now beg to enter into the details of the construction of the present apparatus. The glass disc, the ground surface of which is applied to the upper end of the cylinder, is perforated with three round holes, and that which closes the lower end with only one. A thermometer graduated on its stem, a brass tube with a stop-cock, and another tube also supplied with a stop-cock are fitted through these openings. The thermometer is introduced through a common india-rubber cork, but the other tubes are inserted, air-tight, through studs of vulcanite, so that they cannot possibly be drawn in by atmospheric pressure, while the vacuum is being made in the cylinder. A single-barrel air-pump worked with both hands, while held with the feet, is connected with a brass tube supplied with a stop-cock, fitted through an aperture in the lower glass slab. This tube is also introduced through a stud of vulcanite; but the portion of the stud inside the cylinder is widened out so as to fill up the whole diameter of the cylinder; it is, moreover, slightly funnel-shaped, thus collecting the fluid towards the centre of the bottom of the tube, and allowing it to run out readily through the centre aperture into a small bottle, for subsequent analysis.

The operation is conducted as follows:-The cylinder, well washed and wiped out dry, is placed on its tripod, and, after slightly greasing the rims, the glass slabs with their ground sides in contact with the ground rims of the tube are screwed down upon them above and below. Next, the bag full of the air to be analysed is connected with one of the brass tubes of the upper disc, while a mercury manometer is screwed down tight on the other. The elastic pipe of the airpump is now screwed to the brass tube fitting through the lower glass slab. I now open the cock of the tube in connection with the airpump, while the two others are closed, and extract the air from the cylinder to a pressure of 20 or 30 millimetres, and then the air is let into the cylinder from the bag; this is repeated, and the cylinder becomes perfectly filled with a bulk of air equal to its own capacity. The cylinder I have been using holds 1,993 cubic centimetres. It is important, of course, to take into account the temperature of the air in the bag and in the tube while they are freely in communication with each other. This is easily done, as a thermometer passes into the bag through a neck in which it is fitted air-tight, and another thermometer gives the temperature of the air in the cylinder: both readings are recorded when found to be steady. Hence the bulk of air in the cylinder is measured under exactly the same pressure as that in the bag, and the reduction for the difference of temperature between the air in the bag and tube, if there be any, is easy to calculate.

The stop-cocks are now all closed (that of the tube connected with the air-pump had of course been shut inmediately after making the 
vacuum), and the pump-pipe and manometer are screwed off. I stated in my communications to the Royal Society, that a small pear-shaped vulcanised india-rubber bag had been used as a diverticulum for the air displaced by the introduction of the normal alkaline solution into the cylinder; such a bag was also employed in the present case. It was emptied of its air by being rolled between the fingers, and then its mouth was fitted over the tube to which the manometer had been formerly screwed; on the other hand, a pipette containing 100 c.c. of the normal solution of barium was introduced into the tube originally in communication with the air-bag. The stem of the pipette is served over with thread and wax so as to make it fit air-tight in the tabe, and has a glass stop-cock, checking at will the fall of the solution. The stop-cock of the pipette and of the tube into which it is inserted are now opened, together with that of the tube connected with the diverticulum, and the alkaline solution falls into the cylinder, displacing a certain volume of air which finds its way into the pear-shaped vulcanised india-rubber bag; on grasping the pipette with the hand, after closing its mouth, the heat of the hand drives out the very last drop of the solution, so that the 100 c.c. may be relied upon to fall into the cylinder; the glass cock of the cylinder is then instantly shut.

The brass stop-cocks are now both closed, the empty pipette is taken out of the brass tube, and the cylinder removed from the stand to be agitated gently in a position nearly horizontal. After a minute or so the air in the diverticulum is forced back into the tube by pressure with the fingers, and the cock is closed, preventing any return of air into the small bag. The agitation is resumed, and after a lapse of ten minutes, I have found experimentally that the combination may be considered as complete. It is well to avoid agitating the fiuid in the tube too violently, as such a manipulation appears to interfere with the subsiding of the precipitate of baric carbonate, and creates a diffieulty when it is required to decant a certain bulk of the clear fluid for analysis. Nothing is now left to be done but to open the cocks and draw off the milky liquid into a small bottle holding about 100 c.c., which is immediately corked; the cork is finally dipped into melted paraffin.

The determination of the barinm left in the clear solution, after precipitation of the carbonate, is proceeded with afterwards, certain precautions being taken to be presently stated.

The advantages of the method are manifold.

1st. The whole apparatus is of a portable nature, consisting summarily of a hollow glass cylinder, with its screws, glass slabs, and brass tubes, a mercury manometer, the vulcanised india-rubber diverticulum, a thermometer, a graduated pipette, and the air-pump with

VOL. XXXVII.

$2 \mathrm{~L}$ 
its pipe, plus a light tripod as a stand for the cylinder; it is well to have at hand a spare thermometer and manometer, and a few pipettes, in case of accident.

2nd. The method may be accepted as giving results quite trustworthy, the capacity of the cylinder, together with the correction for temperature, yielding the exact bulk of air to be analysed. The bag containing the air expired is held up by an assistant while the tube is being filled; a certain degree of pressure is thereby exerted from the weight of the bag on the air inside it, having a tendency to increase the amount of air in the cylinder with which the bag is connected. The influence of this slight pressure must be very trifling, and has been neglected; if the correction was required it could be easily made.

3rd. If any doubt be entertained as to the complete combination of the carbonic acid in the cylinder with the barium, it is easy to withdraw a small quantity of air and to test it without detriment to the analysis. This is done by letting air into the india-rubber diverticulum, the stop-cock of the tube with which the diverticulum is connected being opened and then shat; the pear-shaped bag is then withdrawn, care being taken not to let out any air from it, and the sample thus extracted is passed through a solution of baric hydrate; 20 or 30 c.c. of air may thus be removed when the combination is nearly completed, without damaging the analysis. In large cylinders, such as that I have been using, holding 1,993 c.c. of air, the amount of air that can be collected in this way is but small, perhaps too small for testing purposes, because of the diminished pressure inside the cylinder, owing to the large quantity of carbonic acid absorbed by the barium; but this objection would not apply to smaller vessels, such as a cylinder holding about 1,509 c.c. of air, which would be large enough for the analysis of expired air.

The final analysis of the solution of barium can be done at any time, say within a month, and even a longer period, as the cork, covered with paraffin, may be considered practically impervious to air; 25 c.c. of the clear solution, after the precipitate has subsided, are decanted and mixed with 100 c.c. of distilled water ascertained to be free from carbonic acid. The mixture is made in a cylindrical vessel holding but little more than 125 c.c., and which is closed with an india-rubber cork. The fluid is shaken and poured into a 50 c.c. burette, afterwards closed with a cork connected with a tube holding pieces of potassic hydrate, through which air passes into the burette as the fluid is drawn out of it. 5 c.c. of a sclution of oxalic acid of such a strength that 1 c.c. $=1 \mathrm{mgrm} . \mathrm{CO}_{2}$, are measured out into a beaker, and two or three drops of a solution of turmeric added. The amount of fluid from the burette necessary to neutralise the acid is 


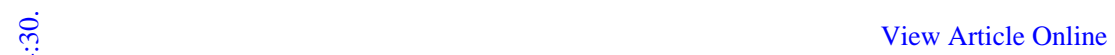

then determined. I have found it necessary, in order to guard against error, to renew frequently the solution of oxalic acid; after a week or ten days, especially in warm weather, the strength of the solution can hardly be relied upon. I have frequently used simultaneously two solutions of oxalic acid made at different times; great accuracy can be obtained by adopting these varions precautions. I prefer the present plan to the dropping of the oxalic acid solution from the burette into a measured volume of the alkaline solution, thus avoiding any error from the carbonic acid present in the atmosphere. As to the time required for the above manipulations, about 25 minutes to half an hour will be wanted, so far as the collection of the milky fluid in the bottle is concerned, and the remainder of the work can be done subsequently at the rate of two or three different analyses per hour.

I subjoin an illustration of the calculations of the analysis which offer no difficulty.

\section{Calculations of the Analysis.}

Volume of the bag full of expired air, 39.3 litres, under 1 inch pressure of water.

Time to fill the bag with expired air, 353 seconds.

Volume of cylinder, 1,993 c.c.

Temperature of air in bag, $60.0^{\circ}$.

Temperature of air in cylinder, $58.0^{\circ}$.

25 c.c. of the clear solution of barium decanted after precipitation and mixed with 100 c.c. of water.

1. 5 c.c. oxalic acid are neutralised by 17.50 c.c. of the mixture.

2.

3.

4.

$"$

$" \quad$,

$17 \cdot 55$

$17 \cdot 60$

$17 \cdot 65$

, ",

\section{Mean..... $\overline{17.57}$}

In many, perhaps most analyses, the four determinations agree closer than in the present case.

$125: 25=17 \cdot 57: x$.

$x=3.514$ pure undiluted solution neatralise 5 c.c. oxalic acid.

$3 \cdot 514: 0: 305 \mathrm{CO}_{2}=100: x$.

$x=0.1423 \quad \mathrm{CO}_{2}$ corresponding to 100 c.c. of the clear solution of bariam.

$\mathrm{CO}_{2}$ corresponding to 100 c.c. normal alkaline solution .... $0 \cdot 2730$

$\mathrm{CO}_{2}$ corresponding to 100 c.c. alkaline solution after precipitation $\ldots \ldots \ldots \ldots \ldots \ldots \ldots \ldots \ldots \ldots \ldots, 0.1423$ 


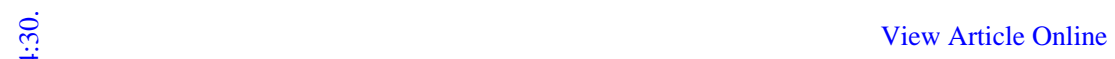

500 GROVES : DETERMINATION OF NITROGEN

Correction of volume of air in cylinder for $2^{\circ} \mathrm{F}$. $=15$ c.c. $1993+15=2008$.

(Litres) $2 \cdot 008: 01307=39 \cdot 3: x$.

$x=2.588$ gram $\mathrm{CO}_{2}$ in the bag.

Seconds. Seconds.

$353: 2 \cdot 558=60: x$.

$x=0.435$ gram $\mathrm{CO}_{2}$ expired per minute.

I have records of upwards of 353 determinations of carbonic acid in air expired under various circumstances, and hope to continue the inquiry. 